\title{
Measurement of Active and Sedentary Behavior in Context of Large Epidemiologic Studies
}

\author{
Charles E. Matthews ${ }^{1}$, Sarah Kozey Keadle ${ }^{1,2}$, Steven C. Moore ${ }^{1}$, Dale S. Schoeller ${ }^{3}$, \\ Raymond J. Carroll ${ }^{4}$, Richard P. Troiano ${ }^{5}$, and Joshua N. Sampson ${ }^{6}$ \\ ${ }^{1}$ Metabolic Epidemiology Branch, Division of Cancer Epidemiology and Genetics, National \\ Cancer Institute, Rockville, MD \\ ${ }^{2}$ Kinesiology Department, California Polytechnic State University, San Luis Obispo, CA \\ ${ }^{3}$ University of Wisconsin, Biotech Center and Nutritional Sciences, Madison, WI \\ ${ }^{4}$ Department of Statistics, Texas A\&M University, College Station, TX and School of Mathematical \\ and Physical Sciences, University of Technology Sydney, Australia \\ ${ }^{5}$ Risk Factor Assessment Branch, Division of Cancer Control and Population Sciences, National \\ Cancer Institute, Rockville, MD \\ ${ }^{6}$ Biostatistics Branch, Division of Cancer Epidemiology and Genetics, National Cancer Institute, \\ Rockville, MD
}

\section{Abstract}

Introduction/Purpose-To assess the utility of measurement methods that may be more accurate and precise than traditional questionnaire-based estimates of habitual physical activity and sedentary behavior we compared the measurement properties of a past year questionnaire (AARP) and more comprehensive measures: an internet-based 24-hour recall (ACT24), and a variety of estimates from an accelerometer (ActiGraph).

Methods-Participants were 932 adults (50-74 y) in a 12-month study that included reference measures of energy expenditure from doubly labeled water (DLW) and active and sedentary time via activPAL.

Results-Accuracy at the group level (mean differences) was generally better for both ACT24 and ActiGraph than the AARP questionnaire. AARP accuracy for energy expenditure ranged from -4 to $-13 \%$ lower than DLW, but its accuracy was poorer for physical activity duration ( $-48 \%)$ and sedentary time $(-18 \%)$ vs. activPAL. In contrast, ACT24 accuracy was within 3 to $10 \%$ of DLW expenditure measures and within 1 to $3 \%$ of active and sedentary time from activPAL. For ActiGraph, accuracy for energy expenditure was best for the Crouter 2-regression method ( -2 to

Corresponding Author: Charles E. Matthews, PhD, Metabolic Epidemiology Branch, Division of Cancer Epidemiology and Genetics, National Cancer Institute, 9609 Medical Center Drive - 6E444, Bethesda, MD 20892-9704, charles.matthews2@nih.gov, Phone: 240-276-7211.

Conflicts of Interest

The authors have no financial conflicts of interest regarding the results of this research.

Results of the present study does not constitute endorsement by ACSM. Results are presented clearly (as possible), honestly, and without fabrication/falsification or with overt data manipulation. 
$-7 \%)$, and for active and sedentary time the 100 count/minute cut-point was most accurate $(-1 \%$ to $2 \%$ ) at the group level. One administration of the AARP questionnaire was significantly correlated with long-term average from the reference measures $\left(\rho_{X \mathrm{~T}}=0.16-0.34\right)$ overall, but 4 ACT24 recalls had higher correlations $\left(\rho_{\mathrm{XT}}=0.48-0.60\right)$, as did 4-days of ActiGraph assessment $\left(\rho_{\mathrm{XT}}=0.54-0.87\right)$.

Conclusion-New exposure assessments suitable for use in large epidemiologic studies (ACT24, ActiGraph) were more accurate and had higher correlations than a traditional questionnaire. Use of better more comprehensive measures in future epidemiologic studies could yield new etiologic discoveries and possibly new opportunities for prevention.

\section{Keywords}

accelerometer; calibration; exercise; measurement error; posture; sitting

\section{INTRODUCTION}

Epidemiologic studies utilizing questionnaire-based measures of habitual moderate-vigorous intensity exercise (e.g. in past year) and highly prevalent sedentary behaviors such as television viewing have provided essential evidence that physical inactivity is associated with increased risk for early mortality, cardiometabolic diseases $(1,2)$, and several cancers (1). While questionnaires have identified many activity-disease associations supporting development of physical activity recommendations (1), they have two basic problems that limit further progress. First, questionnaires are subject to considerable measurement error resulting from the cognitive challenges involved with accurately reporting one's long-term average of complex behaviors that vary substantially within and between days and by season, e.g., $(3,4)$. Second, questionnaires employed in most cohorts do not assess the full spectrum of sedentary behaviors or lower intensity activities of everyday living $(3,5)$; distinct classes of behavior that may have relevant health effects. In prospective studies these two problems result in the underestimation of the strength of the observable associations (attenuation), false negative results (6), and limit our ability to test critical hypotheses about behaviors that are not routinely measured (5).

Rapid development of wearable sensors and mobile communication technologies has dramatically reshaped the potential for measuring human behavior in large-scale epidemiologic studies, possibly offering solutions to these exposure assessment problems. Both accelerometer-based $(7,8)$ and previous day recall instruments $(9,10)$ often have higher validity than questionnaire-based measures of these behaviors (11), and both methods can measure the full spectrum of daily activities. Taking advantage of new technologies to apply better measures in future studies could lead to stronger associations and less controversy, expansion of our already extensive evidence-base, and ultimately fresh opportunities for prevention. However, the improvements in fidelity associated with these new measures for estimating long-term average behaviors compared to questionnaires is uncertain, and differences in the measurement properties between accelerometer-based measures and previous-day recalls are not well characterized. 
Thus, our primary objective was to examine the measurement properties of a past year questionnaire; an internet-based previous day recall; and measures derived from the ActiGraph accelerometer-all compared to high quality reference measures of energy expenditure (12) and sedentary and physically active time from activPAL. A secondary objective was to describe the measurement properties of historically relevant ActiGraph calibration methods.

\section{METHODS}

\section{Study Design}

The Interactive $\underline{\text { Diet and }} \underline{\mathbf{A}}$ ctivity $\underline{\text { Tracking in }} \underline{\mathbf{A}} \mathrm{ARP}$ (iDATA) study was designed to evaluate a variety of diet and physical activity measures suitable for use in epidemiologic studies. Participants were a convenience sample of AARP members (50-74 years) from Pittsburgh, PA who spoke English, had internet access, were not on a weight-loss diet, had a body mass index (BMI) $<40 \mathrm{~kg} / \mathrm{m}^{2}$, and were free of major medical conditions and mobility limitations. Initial contact was made via automated phone call and invitation letter directing those interested to contact the study (see Figure, SDC 1, Participant recruitment). The study was approved by the NCI Special Studies Institutional Review Board. All participants signed informed consent and those who completed the full study received $\$ 450$. Participants visited the study center up to 3 times over 12-months and completed several diet and physical activity measurements. Figure 1 shows the physical activity measurement schedule for group one (see Tables for full measurement schedule, SDC 2, Schedule for Group 1; SDC 3, Schedule for all Groups). A more extensive description of our methods is provided elsewhere (see Text, SDC 4, Detailed Methods).

\section{Reference Measures}

\section{Doubly Labeled Water (DLW) and Resting Energy Expenditure (REE)-Total} energy expenditure (TEE) was measured via DLW (12) using established methods $(13,14)$. The method is accurate compared to indirect calorimetry $( \pm 2 \%)$ with an individual level precision of 5 to $7 \%$. REE pred was estimated using the Mifflin-St. Jeor equation (15), developed on 498 adults (19-78 years; $\left.\mathrm{R}^{2}=0.71\right)$. It has been cross-validated in men and women (20-78 yrs) classifying $82 \%$ of non-obese adults within $10 \%$ of measured REE (16). Thirty-two participants repeated TEE and $\mathrm{REE}_{\text {pred }}$ measures (6-months apart).

activPAL - activPAL 3D (PAL Technologies, Glasgow, SCT) is an accelerometer worn on the mid-right thigh. It estimates time spent lying, sitting, standing, stepping, and non-wear time. Participants were asked to wear it continuously (24-hours) for 7 consecutive days and to record the date/time they got out of bed in the morning and into bed each night to determine the "waking day". Sedentary behavior was estimated as time sitting or lying during the waking day, and active time as the sum of standing and stepping, consistent with recent Sedentary Behavior Research Network definitions/concepts (17). This approach assumes that behaviors done while standing are in the physically active category. Accuracy for posture in laboratory settings is $90 \%-100 \%$ (18), and there is strong agreement $\left(\mathrm{R}^{2}=0.94-0.96\right)$ vs. direct observation in free-living studies $(10,19)$. Two 7-day administrations were obtained six-months apart (Figure 1). 


\section{Test Measures}

\section{AARP Physical Activity and Sedentary Behavior Questionnaire-This}

questionnaire asked, during the past 12 months, how much time participants spent in sleep, physical activity and sitting. The physical activity questions include 8 items focused on exercise and sports that have been evaluated previously (20), and 8 focused on non-exercise activities (e.g., household chores, lawn and garden, daily walking; see Figure, SDC 5, AARP Physical Activity Questionnaire). Sitting and non-exercise portions of the survey have not been evaluated previously, but have been associated with mortality (21). The AARP survey was administered twice, 6-months apart (Figure 1).

Activities Completed Over Time in 24 Hours (ACT24)_ACT24 is an internet-based previous-day recall designed to estimate total time (hrs/d) spent sleeping (in bed), sedentary, and in physical activity, and to estimate energy expenditure associated with these behaviors (MET-hrs/d). Building on content and methods developed and refined previously $(6,10)$, we developed a self-administered internet-based recall that captures details about individual behaviors over 24-hours. To complete ACT24, respondents select from more than 200 individual activities, record the duration of each, and answer pertinent follow-up questions including body position (see Image, SDC 6, ACT24 Screen-shot). Sedentary behaviors were defined as those performed during the waking day (out of bed) while sitting or reclining and that require little energy expenditure (typically < 1.8 METs). In contrast, active behaviors were those involving an upright posture, or performed in any posture with higher MET levels. Participants were cued via email to complete up to six recalls during the 12-month study, one recall every other month (Figure 1). Further ACT24 information is available on request.

ActiGraph Measures-ActiGraph (model GT3X) is a triaxial accelerometer and participants were asked to wear the monitor on the right hip for 7-days from the time they got out of bed for the day until they went to bed for the night. It was initialized to record triaxial acceleration in 1-s epochs with the low-frequency extension enabled. On/off logs were used to confirm wear dates, estimate the waking day, and the Choi algorithm was used to estimate non-wear time during this period. We selected scoring methods reflecting the evolution of the underlying ActiGraph technology and its calibration methods over last 20 years (22-27). See Table, SDC 7, Detailed description of ActiGraph calibration methods). Two expenditure prediction equations were derived from calibration studies that used ambulatory activities to develop linear equations from 60-second epoch acceleration signals from the vertical (Freedson, (22)) or 3-dimensional axes (Sasaki, (23)). Two equations were calibrated to a broad range of activities and used shorter epochs and more sophisticated modeling. The Crouter method (24) applies two-regression equations developed from 18 activities to estimate energy expenditure from activity counts (vertical axis). Sojourn 3x (Soj3x) (25) employs three-dimensional acceleration features from 1 second epochs and hybrid machine-learning and neural networks trained on approximately 30 activities (28) to classify behaviors and estimate energy expenditure. Sedentary and active time was estimated from Crouter and Soj3x and two cut-point methods; 100 counts/min vertical axis (100VT; (26)) and 200 counts/min, vector magnitude (200VM) (27). We also calculated time spent in non-sedentary light ( $<3$ METs) and moderate-vigorous intensity activity (3+ METs) using 
the above methods, and common cut-points $(22,29)$. Two 7-day ActiGraph administrations were obtained six-months (Figure 1).

\section{Accounting for Monitor Non-wear and Missing Time on Self-Report Instruments}

To minimize differences between measures due to non-wear time during the waking day, we imputed values for this time on valid monitor days. During the mean $15.8 \mathrm{hrs} / \mathrm{d}$ waking day reported, activPAL recorded 0.7 non-wear hrs/d, while the ActiGraph recorded 1.9 non-wear hrs/d. For valid activPAL and ActiGraph days, we imputed values for active and sedentary time and energy expenditure in proportion to time recorded in sedentary, light, and moderate-vigorous intensity activity during the waking day for each monitor. ACT24 missing time was minimal $(0.005 \mathrm{hrs} / \mathrm{d})$, so no adjustments were made. Missing time on the AARP Questionnaire was so large $(6.2 \mathrm{hrs} / \mathrm{d})$ that we felt there was inadequate information for meaningful imputation. See Figure, SDC 8, Description of imputation results.

\section{Calculation of Indices for Total and Physical Activity Energy Expenditure}

We calculated TEE from the test measures using their energy cost estimates (MET-hrs) for time in bed (sleeping), sedentary, and in physical activity using REE $_{\text {pred }}$ from the Mifflin-St Jeor equation, including an addition of 10\% of TEE for the thermic effect of food. For all measures, PAEE was calculated as PAEE $=(\mathrm{TEE} \times 0.9)-\mathrm{REE}_{\text {pred }}$; physical activity index $(\mathrm{PAI})$ as $\mathrm{PAI}=\mathrm{PAEE} /$ body mass (30). We also calculated physical activity levels (PAL=TEE/ REE $\left._{\text {pred }}\right)$.

\section{Statistical Methods}

First, for our reference measures we described the mean (SD) of their summary metrics and evaluated their variation over 6-months using correlations. Second, to assess the overall accuracy of our test measures at the group level we calculated the mean (SD) of all available data for each measure and then calculated mean differences (SD) between these values and our reference measures (DLW, 1 administration; activPAL, mean of all valid days) and conducted t-tests of these differences. We then modeled the error structure of each test instrument using the replicate measures of each (i.e., replicate administrations of AARP and replicate days of observation for ACT24 and ActiGraph) while accounting for intraindividual variation in our reference measures. To do so, we assumed each instrument's objective was to estimate the long-term (yearly) average of the summary metric (e.g. PAEE). Let $T_{i}$ be the true long term average. Then, we modeled the test instrument's value of that metric at time $j$ as $Q_{i j}$,

$$
Q_{i j}=\beta_{0}+\beta_{1} T_{i}+R_{i}+\varepsilon_{i j}
$$

where $T_{i} \sim N\left(\mu_{T}, \sigma_{T}^{2}\right)$ is a random variable representing the true yearly average, $R_{i} \sim N\left(0, \sigma_{R}^{2}\right)$ is a random variable representing person-specific bias and $\varepsilon_{i j} \sim N\left(0, \sigma_{\varepsilon}^{2}\right)$ is a random variable representing intra-individual variability and measurement error. We then estimated model parameters: bias $\left(\beta_{0}\right)$, slope $\left(\beta_{1}\right)$, and relevant variances including total 
variance ( $V=\beta_{1}^{2} \sigma_{T}^{2}+\sigma_{R}^{2}+\sigma_{\varepsilon}^{2}$ ), and the percent of variance attributable to person-specific bias $\left(P_{R}=100 \sigma_{R}^{2} / V\right)$ and random error $\left(P_{\varepsilon}=100 \sigma_{\varepsilon}^{2} / V\right)$.

We estimated these parameters by incorporating information from the reference instruments. We modeled the reference instrument's value at time $j$ as $F_{i j}$

$$
F_{i j}=T_{i}+U_{i j}
$$

where $U_{i j} \sim N\left(0, \sigma_{U}^{2}\right)$ is a random variable representing intra-individual variability and measurement error. Then the combined data follows a multivariate normal described by equations (1) and (2). For example, if we consider DLW and the AARP questionnaire as the reference and test instruments, the data for an individual with two measures from each instrument would follow equation (3)

$\left[\begin{array}{c}Q_{i 1} \\ Q_{i 2} \\ F_{i 1} \\ F_{i 2}\end{array}\right] \sim N\left(\left[\begin{array}{c}\beta_{0}+\beta_{1} \mu_{T} \\ \beta_{0}+\beta_{1} \mu_{T} \\ \mu_{T} \\ \mu_{T}\end{array}\right],\left[\begin{array}{cccc}\sigma_{r}^{2}+\sigma_{e}^{2}+\beta_{1}^{2} \sigma_{T}^{2} & \sigma_{r}^{2}+\beta_{1}^{2} \sigma_{T}^{2} & \beta_{1} \sigma_{T}^{2} & \beta_{1} \sigma_{T}^{2} \\ \sigma_{r}^{2}+\beta_{1}^{2} \sigma_{T}^{2} & \sigma_{r}^{2}+\sigma_{e}^{2}+\beta_{1}^{2} \sigma_{T}^{2} & \beta_{1} \sigma_{T}^{2} & \beta_{1} \sigma_{T}^{2} \\ \beta_{1} \sigma_{T}^{2} & \beta_{1} \sigma_{T}^{2} & \sigma_{T}^{2}+\sigma_{U}^{2} & \sigma_{T}^{2} \\ \beta_{1} \sigma_{T}^{2} & \beta_{1} \sigma_{T}^{2} & \sigma_{T}^{2} & \sigma_{T}^{2}+\sigma_{U}^{2}\end{array}\right]\right)_{(3)}$

We can obtain estimates by maximizing the complete likelihood (over all subjects and replicate measures) using the mle function in $\mathrm{R}$ and obtain standard errors by the bootstrap procedure. When the test and reference instrument occurred during overlapping time periods (i.e., same day of activPAL and ActiGraph measures), we allowed the error terms, $U_{i j}{ }^{\prime}$ and $\varepsilon_{i j}$, to be correlated. Note, this approach incorporates the sub-group with replicate DLW measures and the number of measures per subject can differ across subjects. We then estimated the correlation, $\rho_{T X}$, between $X$ replicates of the test instruments (i.e., number of AARP administrations or days of ACT24 and ActiGraph), and the true yearly average

$$
\rho_{T X}=\frac{\beta_{1} \sigma_{T}^{2}}{\sqrt{\left(\beta_{1}^{2} \sigma_{T}^{2}+\sigma_{R}^{2}+\sigma_{\varepsilon}^{2} / X\right) \sigma_{T}^{2}}}
$$

and we estimated the corresponding attenuation factor, $\lambda_{X}$,

$$
\lambda_{X}=\frac{\beta_{1}}{\beta_{1}^{2}+\sigma_{R}^{2} / \sigma_{T}^{2}+\left(\sigma_{\varepsilon}^{2} / X\right) / \sigma_{T}^{2}} .
$$

As $X$ increases, random error is reduced and the correlation and the attenuation factors increase.

\section{RESULTS}

A total of 932 participants completed baseline measures and had valid reference measures for TEE ( $n=689$ ) or activPAL ( $n=932$ ), see Table 1. From 9,553 valid activPAL days we had 
a mean of 10.3 days/participant. There were 5,009 valid ACT24 recalls (5.4 recalls/ participant $(\mathrm{SD}=1.1)$ ), 24.2 episodes $(\mathrm{SD}=7.9)$ of sedentary and active behaviors per recall, and median completion time was 30 minutes. There were 8,731 valid ActiGraph days, and a mean of $9.3(\mathrm{SD}=3.5)$ days per participant. Evaluation of intra-individual variability over 6months in our reference instruments revealed TEE from DLW to be relatively stable for all participants $(\mathrm{r}=0.81)$, but physical activity indices were more variable $(\mathrm{r}=0.57-0.65$; see Table, SDC 9, Six-month correlations). Using the mean of valid days in each period, activPAL stability was $r=0.62$ to 0.65 overall. Stability was marginally higher in men than women for both reference measures.

Average accuracy (mean differences) of TEE reported on AARP and ACT24 were similar to DLW (within 3-4\%), while ActiGraph TEE measures ranged from $-21 \%$ to $-2 \%$ lower than DLW (Table 2). In terms of PAEE, PAL, and PAI, accuracy for AARP and ACT24 was often similar, but AARP underestimated activity duration by $48 \%$ and sedentary time by $18 \%$, while ACT24 was within 1-3\% of activPAL values. The most accurate ActiGraph expenditure estimates were from Crouter, while 100VT was most accurate for active and sedentary time. Overall, the accuracy for ACT 24 and ActiGraph measures were better than AARP, and ACT24 and ActiGraph methods were often comparable. We also examined accuracy by sex and BMI and did not find large consistent differences in both reference measures (see Tables, SDC 10, Mean differences by sex; SDC 11, Mean differences by BMI; SDC 12, Correlations in all participants and by sex).

Next, using measurement error models we evaluated the ability of test instruments to estimate the yearly average of each metric (Table 3 ). Comparing self-report methods across all metrics, the AARP questionnaire had flatter slope terms reflecting larger amounts of systematic error (i.e., less sensitivity) than did ACT24, particularly for active and sedentary time. ACT24 also had less person-specific bias than AARP, but a single ACT24 recall usually had more random error. Better results, larger slope terms and less person-specific bias and random error, were also noted for ActiGraph in comparison to AARP. ACT24 and ActiGraph often had comparable slope terms (except for PAL) and person-specific bias, but random error was greater for ACT24.

We then evaluated correlations between the long-term average from our reference measures and a given number of replicates of each test instrument. Although one administration (replicate) of AARP had significant positive correlations for physical activity and sedentary behavior overall $\left(\rho_{\mathrm{TX}}=0.16-0.34\right.$; see Table $\mathrm{C}$, just 1 day (replicate) of ACT24 or ActiGraph had equal or higher correlations $\left(\rho_{\mathrm{TX}}=0.33-0.66\right)$. Four ACT24 recalls had substantially higher correlations overall $\left(\rho_{\mathrm{TX}}=0.48-0.60\right)$, as did 4 days of ActiGraph $\left(\rho_{\mathrm{TX}}=0.54-0.87\right)$. There were differences in patterns of correlation in men and women (Figure 2). For expenditure metrics, AARP correlations were higher in men than women, while for ACT24 and ActiGraph the reverse was true. Among women there was more variation in strength of the correlations between ACT24 and ActiGraph, with device-based measures having higher correlations than ACT24, but there were minimal differences in men. Comparing strength of the correlations between older simpler ActiGraph calibration methods (Freedson, 100VT) to newer and more sophisticated methods (Crouter, Soj3x), the correlations were modestly 
stronger in women for newer methods, but differences in correlation strength between older and newer methods were not evident in men.

Next, to describe the impact of measurement error induced attenuation in the context of an epidemiologic study we calculated attenuation factors $\left(\lambda_{X}\right.$; see Table, SDC 13, Attenuation factors by sex) and the associated reduction in observable relative risk, assuming a true RR of 4.0 (i.e., $\mathrm{RR}_{\mathrm{obs}}=4.0^{\lambda x}$ ). Sex differences here were generally small and inconsistent across test instruments, so we present results for all participants (Figure 3). A single administration (replicate) of the AARP questionnaire would find $\mathrm{RR}_{\mathrm{obs}}=1.36-1.38$ for physical activity and only an $R_{\mathrm{obs}}=1.10$ for sedentary time when the true association was 4.0. In contrast, 4 ACT24 replicates would find $\mathrm{RR}_{\mathrm{obs}}=1.84-1.87$ for both physical activity and sedentary time, while 4 ActiGraph replicates would find $R_{\mathrm{obs}}=2.03-2.64$. Freedson PAEE estimates unexpectedly produced attenuation factors greater than 1.0 due to flattened slope terms and total variance values that were lower than the DLW measures, resulting in $R_{\mathrm{obs}}>4.0$ (Figure 2, top panel).

To evaluate light and moderate-vigorous intensity activity (hrs/d) from AARP and ACT24 we made comparisons to Soj3x by averaging all available data for each instrument. For light and moderate-vigorous activity mean values were: Soj3x, 4.58 and $1.96 \mathrm{hrs} / \mathrm{d}$; AARP, 1.13 and $1.97 \mathrm{hrs} / \mathrm{d}$; and ACT24, 4.19 and $1.93 \mathrm{hrs} / \mathrm{d}$, respectively (see also SDC Figure 2). Pearson correlations with Soj3x were lower for AARP (Light, $r=0.23$; moderate-vigorous, $\mathrm{r}=0.25$ ) compared to ACT24 (Light, $\mathrm{r}=0.34$; moderate-vigorous, $\mathrm{r}=0.47$ ).

\section{DISCUSSION}

In this free-living evaluation of three distinct exposure assessment methods suitable for use in large epidemiologic studies, we found evidence of significant correlations for a past-year questionnaire (AARP), but this instrument underestimated physical activity duration by $48 \%$ and sedentary time by $18 \%$. In comparison, an internet-based previous-day recall (ACT24) and an accelerometer (ActiGraph) provided better accuracy, perhaps because of their more complete coverage of common daily activities, and these instruments also were more strongly correlated with the reference measures. In addition, the lower measurement error in ACT24 and ActiGraph were predicted to result in less attenuation in observed risk estimates compared to the AARP questionnaire. Notably, ACT24 often had correlations of similar strength as ActiGraph, although the accelerometer was predicted to produce less attenuated risk estimates. Overall, these findings suggest that either ACT24 or accelerometer-based measures of long-term average behavior may overcome the two major limitations of traditional physical activity questionnaires. Below, we discuss findings for our self-report measures (AARP, ACT24), the ActiGraph, and then all methods in context of possible application in future epidemiologic studies.

Questionnaires which rely on long-term recall to assess habitual behaviors have been the mainstay of physical activity epidemiology, and their weaknesses have been widely discussed (e.g., $(4,5))$. Nevertheless, consistent with previous studies $(5,31-33)$ the AARP questionnaire demonstrated some utility for ranking participants, with significant correlations ranging from 0.33 for PAEE to 0.16 for sedentary time with one administration. 
However, the limited accuracy at the group level for total physical activity duration (-48\%) and sitting time (-18\%) suggests incomplete assessment of these behaviors and points to challenges in translating etiologic associations derived from this tool to public health practice. Two studies recently evaluated more comprehensive physical activity and sedentary time questionnaire and reported marginally higher correlations with PAEE from DLW of $\mathrm{r}=0.40$ (34) and $\mathrm{r}=0.46$ (32), and a correlation of $\mathrm{r}=0.27$ for sedentary time vs. accelerometer (32). Correlations for the AARP-based measures of sedentary time were lower but in-line with those derived from a single sitting time question vs. activPAL (35). In contrast, ACT24 had minimal bias $(<10 \%)$ and just 4 replicates had higher correlations for PAEE $(r=0.53)$, active time $(\mathrm{r}=0.58)$, and sedentary time $(\mathrm{r}=0.60)$ than the AARP survey. These findings are in-line with other previous day recall studies in adults $(10,36)$. Overall, our findings suggest that ACT24 can produce estimates of physical activity and sedentary time that have less bias and are more strongly correlated with strong reference measures than traditional questionnaires, presumably because it is more accurate for participants to report what they did yesterday than what they did last year.

Our results for ActiGraph were also stronger than those for AARP, although there were substantial differences in accuracy of the monitor-based estimates depending on the processing/calibration method evaluated. Otherwise, our ActiGraph results for rank-ordering participants (correlations) were largely consistent with previous studies. They also suggest that newer calibration methods provided modest improvements over earlier methods for this purpose. Jeran and colleagues (37) systematically reviewed 19 accelerometer-DLW studies and reported a median correlation for PAEE of $r=0.50$, primarily from first generation accelerometers using simple summary metrics (e.g., activity counts). Here, we focus on correlations for 4 replicate days of monitor wear for comparability with previously discussed ACT24 results. Evaluating PAEE, the Crouter (24) and Soj3x (25) methods calibrated with 18 to 30 and activities of everyday living including walking and running, coupled with more sophisticated prediction models had modestly stronger correlations $(r=0.64-0.65)$ than methods which employed only linear models derived from 3 ambulatory activities (Freedson, $r=0.59$; Sasaki, $r=0.54$ ). There was a suggestion of marginally higher correlations for active and sedentary time for methods that used 3-dimensional acceleration signals (Sasaki, Soj3x) compared to methods using vertical acceleration (100VT, Crouter). Both of these findings are consistent with Lyden (25) who used direct observation as a reference. Similarly, Kim (38) reported validity estimates (phi) for sedentary time of 0.89, 0.72, 0.61, and 0.54 for activPAL, Soj3x, 100VT, and Crouter, respectively using a wearable camera for reference. Newer movement metrics derived from raw acceleration signals compared to the activity count-based methods we used here have shown better performance in a laboratory studies (e.g., (39)), but rigorous testing of these methods in free-living study samples vs. strong reference measures remains limited, making comparison to this study difficult. Interestingly, we found that the most sophisticated calibration/modeling method (Soj3x) had modestly higher correlations than simpler methods in women, but not in men. Reasons for this are not clear, but it may be that the suite of activities employed by Soj3x was a better match for the daily activities of women than men. Additional research is needed to confirm and better understand this finding, and to understand the potential improvements associated with modeling raw acceleration data. 
Studies that have employed measurement error models to estimate attenuation factors for questionnaires are relatively few, and variation in the questionnaires tested, measurement error models fit, and the reference measures employed makes comparison challenging. To simplify comparison between previous studies and this one, we calculate $R_{\mathrm{obs}}$ for a true $\mathrm{RR}$ of 4.0 using attenuation factors reported in previous studies (i.e., $\mathrm{RR}_{\mathrm{obs}}=4.0^{\lambda}$ ). Spiegelman (40) compared a past year questionnaire to 28-days of physical activity diaries and reported results consistent with $\mathrm{RR}_{\mathrm{obs}}=1.52(\lambda=0.30)$, while Ferrari (41) compared a past year questionnaire to both activity diaries and an accelerometer using structural equation modeling and reported results consistent with a $\mathrm{RR}_{\mathrm{obs}}=1.20(\lambda=0.13)$. These findings are in line with the current study. In contrast, Tooze (33) compared a questionnaire that assessed the last 30 days to PAL measured by DLW and reported data consistent with $\mathrm{RR}_{\mathrm{obs}}=2.75(\lambda=0.73)$ in men and an $\mathrm{RR}_{\mathrm{obs}}=1.82(\lambda=0.43)$ in women. It may be that shorter recall periods (30-days) are reported more accurately than longer recall periods of one year. Collectively, these findings and our results suggest that either ACT24 or ActiGraph measures can identify stronger true associations than past year questionnaires.

Decisions about whether ACT24 or an accelerometer are optimal for future studies depend on the etiologic question of interest and the resources available to administer the measures in large populations. Such studies can be very large (e.g., N=200,000) and often strive to enroll participants in a short time-period (e.g., 3 years). In this context, rates of accrual can exceed 1,000 participants per week, which requires a substantial allocation of study resources to complete accelerometer-based measures in all participants. While ACT24 has better measurement properties than many questionnaires and would provide greater contextual detail about specific types of activity than ActiGraph, the more sophisticated accelerometer measures had less overall error and would provide more granular time-stamped data. Accelerometers are being implemented in increasingly large studies such as the UK Biobank ( $n \sim 100,000$ ), but the costs of administering devices in such studies can still be a major challenge and ACT2 4 could be a less expensive alternative. The present study demonstrated that it is possible to collect large numbers of high quality self-administered previous-day recalls using ACT24, but additional research is needed to refine and optimize its application in larger prospective studies.

This study had several strengths and limitations. Its strengths include a large sample-size and a 12-month study period, use of two strong reference measures which largely provided convergent results, evaluation of two different approaches to self-report and a variety of ActiGraph data analysis methods specifically selected to represent evolution of the device over 20 years. Although our modeling approach accounted for intra-individual variation in our reference measures and allowed us to test measures on a per replicate basis, the modest sample-size for the DLW replicates could have reduced our precision of the intra-individual variation in these measures. Additionally, we imputed non-wear time during the waking day so that differences observed between instruments would reflect the underlying measurement properties of each instrument, rather than differences in missing/non-wear time. See SDC Figure 2 for comparison of the original and adjusted mean duration values. Imputation had minimal impact on the correlation and attenuation results. Further limitations include use of: a convenience sample of healthy mostly non-obese adults; only one physical activity questionnaire to assess past year behavior; and only one type of accelerometer worn on the 
waist that did not collect raw acceleration data. Also, our comparisons of activPAL and ActiGraph were for total duration of sedentary and physically active time accumulated over the day. It may be that the pattern of accumulation as measured by each instrument was more different than our results for total duration indicates. Last, the reference measure (Soj3x) we used to evaluate ACT24 and AARP for light and moderate-vigorous intensity activity has only limited validity information for these metrics (25), so our results should be interpreted accordingly.

In conclusion, in this large study evaluating three different methods for measuring physical activity and sedentary behavior we found evidence that the AARP questionnaire can rank order participants but that physically active and sedentary time were substantially underestimated, perhaps due to incomplete coverage of these behaviors. In contrast, replicate measures of a previous-day recall (ACT24) and an accelerometer (ActiGraph) provided more accurate and thus more comprehensive estimates of these behaviors and had higher correlations and attenuation factors indicating less overall measurement error. This study provides important new evidence that new improved measures can overcome the two main limitations of traditional questionnaires that limit further progress in our field. Application of these new methods could lead to key scientific advances that would inform future public health recommendations.

\title{
Supplementary Material
}

Refer to Web version on PubMed Central for supplementary material.

\section{Acknowledgments}

\author{
Support and Funding \\ CEM, SCM, JNS were supported by the NIH/NCI Intramural Research Program; SKK was supported by NCI's \\ Cancer Prevention Fellowship Program; RJC was supported by a grant from the National Cancer Institute (U01- \\ CA057030); DSS was supported by a grant from the National Cancer Institute (RR020915).

\section{Thank You} \\ The authors would like to thank the many NCI investigators involved in the design and conduct of the iDATA study \\ including its PI's - Heather Bowles (Division of Cancer Prevention [DCP]) and Yikyung Park (now Washington \\ University), as well as Amy Subar (Division of Cancer Control and Population Sciences [DCCPS]), Victor Kipnis \\ (DCP), Kevin Dodd (DCP), Douglas Midthune (DCP), and the late Arthur Schatzkin (Division of Cancer \\ Epidemiology and Genetics). We also thank colleagues at Westat, Inc., including: Beth Mittl, Ann Truelove, Chris \\ Bingley for ACT24 development/management and Kerry Grace Morrissey and Susan Yurgalevitch for iDATA \\ project management and field operations/data collection.
}

\section{References}

1. Physical Activity Guidelines Advisory Committee. Physical Activity Guidelines Advisory Committee Report. Washington, DC: 2008. 2008. Report No

2. Biswas A, Oh PI, Faulkner GE, et al. Sedentary time and its association with risk for disease incidence, mortality, and hospitalization in adults: A systematic review and meta-analysis. Ann Intern Med. 2015; 162(2):123-32. [PubMed: 25599350]

3. Matthews CE, Moore SC, George SM, Sampson J, Bowles HR. Improving Self-reports of Active and Sedentary Behaviors in Large Epidemiologic Studies. Exerc Sport Sci Rev. 2012; 40(3):118-26. [PubMed: 22653275] 
4. Shephard RJ. Limits to the measurement of habitual physical activity by questionnaires. Br J Sports Med. 2003; 37(3):197-206. [PubMed: 12782543]

5. Neilson HK, Robson PJ, Friedenreich CM, Csizmadi I. Estimating activity energy expenditure: how valid are physical activity questionnaires? Am J Clin Nutr. 2008; 87(2):279-91. [PubMed: 18258615]

6. Schatzkin A, Subar AF, Moore S, Park Y, Potischman N, Thompson FE, et al. Observational Epidemiologic Studies of Nutrition and Cancer: The Next Generation (with Better Observation). Cancer Epidemiol Biomarkers Prev. 2009; 18(4):1026-32. [PubMed: 19336550]

7. Hills AP, Mokhtar N, Byrne NM. Assessment of Physical Activity and Energy Expenditure: An Overview of Objective Measures. Front Nutr. 2014; 1:5. [PubMed: 25988109]

8. Atkin AJ, Gorely T, Clemes SA, Yates T, Edwardson C, Brage S, et al. Methods of Measurement in epidemiology: Sedentary Behaviour. Int J Epidemiol. 2012; 41(5):1460-71. [PubMed: 23045206]

9. Gomersall SR, Olds TS, Ridley K. Development and evaluation of an adult use-of-time instrument with an energy expenditure focus. J Sci Med Sport. 2011; 14(2):143-8. [PubMed: 20932797]

10. Matthews CE, Keadle SK, Sampson J, Lyden K, Bowles HR, Moore SC, et al. Validation of a previous-day recall measure of active and sedentary behaviors. Med Sci Sports Exerc. 2013; 45(8): 1629-38. [PubMed: 23863547]

11. Helmerhorst HJ, Brage S, Warren J, Besson H, Ekelund U. A systematic review of reliability and objective criterion-related validity of physical activity questionnaires. Int J Behav Nutr Phys Act. 2012; 9(103):1-55. [PubMed: 22233712]

12. Schoeller DA, van Santen E. Measurement of energy expenditure in humans by doubly labeled water method. J Appl Physiol. 1982; 53(4):955-9. [PubMed: 6759491]

13. Trabulsi J, Troiano RP, Subar AF, Sharbaugh C, Kipnis V, Schatzkin A, et al. Precision of the doubly labeled water method in a large-scale application: evaluation of a streamlined-dosing protocol in the Observing Protein and Energy Nutrition (OPEN) study. Eur J Clin Nutr. 2003; 57(11):1370-7. [PubMed: 14576749]

14. Schoeller DA, Ravussin E, Schutz Y, Acheson KJ, Baertschi P, Jequier E. Energy expenditure by doubly labeled water: validation in humans and proposed calculation. Am J Physiol. 1986; 250(5 Pt 2):R823-30. [PubMed: 3085521]

15. Mifflin MD, St Jeor ST, Hill LA, Scott BJ, Daugherty SA, Koh YO. A new predictive equation for resting energy expenditure in healthy individuals. Am J Clin Nutr. 1990; 51(2):241-7. [PubMed: 2305711]

16. Frankenfield DC, Rowe WA, Smith JS, Cooney RN. Validation of several established equations for resting metabolic rate in obese and nonobese people. J Am Diet Assoc. 2003; 103(9):1152-9. [PubMed: 12963943]

17. Tremblay MS, Aubert S, Barnes JD, Saunders TJ, Carson V, Latimer-Cheung AE, et al. Sedentary Behavior Research Network (SBRN) - Terminology Consensus Project process and outcome. Int J Behav Nutr Phys Act. 2017; 14(1):75. [PubMed: 28599680]

18. Edwardson CL, Rowlands AV, Bunnewell S, Sanders J, Esliger DW, Gorely T, et al. Accuracy of Posture Allocation Algorithms for Thigh- and Waist-Worn Accelerometers. Med Sci Sports Exerc. 2016; 48(6):1085-90. [PubMed: 26741122]

19. Lyden K, Kozey Keadle SL, Staudenmayer JW, Freedson PS. Validity of two wearable monitors to estimate breaks from sedentary time. Med Sci Sports Exerc. 2012; 44(11):2243-52. [PubMed: 22648343]

20. Wolf AM, Hunter DJ, Colditz GA, Manson JE, Stampfer MJ, Corsano KA, et al. Reproducibility and validity of a self-administered physical activity questionnaire. Int J Epidemiol. 1994; 23(5): 991-9. [PubMed: 7860180]

21. Matthews CE, Moore SC, Sampson J, Blair A, Xiao Q, Keadle SK, et al. Mortality Benefits for Replacing Sitting Time with Different Physical Activities. Med Sci Sports Exerc. 2015; 47(9): 1833-40. [PubMed: 25628179]

22. Freedson PS, Melanson E, Sirard J. Calibration of the Computer Science and Applications, Inc. accelerometer. Med Sci Sports Exerc. 1998; 30:777-81. [PubMed: 9588623]

23. Sasaki JE, John D, Freedson PS. Validation and comparison of ActiGraph activity monitors. J Sci Med Sport. 2011; 14(5):411-6. [PubMed: 21616714] 
24. Crouter SE, Kuffell E, Hass JD, Frongillo EA, Bassett DR. Refined Two-Regression Model for the ActiGraph Accelerometer [Article]. Med Sci Sports Exerc. 2010; 42(5):1029-37. [PubMed: 20400882]

25. Lyden K, Keadle SK, Staudenmayer J, Freedson PS. A method to estimate free-living active and sedentary behavior from an accelerometer. Med Sci Sports Exerc. 2014; 46(2):386-97. [PubMed: 23860415]

26. Matthews CE, Chen KY, Freedson PS, Buchowski MS, Beech BM, Pate RR, et al. Amount of time spent in sedentary behaviors - United States 2003-2004. Am J Epidemiol. 2008; 167:875-81. [PubMed: 18303006]

27. Aguilar-Farias N, Brown WJ, Peeters GM. ActiGraph GT3X+ cut-points for identifying sedentary behaviour in older adults in free-living environments. J Sci Med Sport. 2014; 17(3):293-9.

[PubMed: 23932934]

28. Freedson PS, Lyden K, Kozey-Keadle S, Staudenmayer J. Evaluation of artificial neural network algorithms for predicting METs and activity type from accelerometer data: validation on an independent sample. J Appl Physiol. 2011; 111(6):1804-12. [PubMed: 21885802]

29. Matthews CE. Calibration of Accelerometer Output for Adults. Med Sci Sports Exerc. 2005; 37(11):S512-S22. [PubMed: 16294114]

30. Schoeller DA, Jefford G. Determinants of the energy costs of light activities: inferences for interpreting doubly labeled water data. Int J Obes Relat Metab Disord. 2002; 26(1):97-101. [PubMed: 11791153]

31. Healy GN, Clark B, Winkler EAH, Gardiner PA, Brown WJ, Matthews CE. Measurement of Adults' Sedentary Time in Population-Based Studies. Am J Prev Med. 2011; 41(2):216-27. [PubMed: 21767730]

32. Besson H, Brage S, Jakes RW, Ekelund U, Wareham NJ. Estimating physical activity energy expenditure, sedentary time, and physical activity intensity by self-report in adults. Am J Clin Nutr. 2010; 91(1):106-14. [PubMed: 19889820]

33. Tooze JA, Troiano RP, Carroll RJ, Moshfegh AJ, Freedman LS. A measurement error model for physical activity level as measured by a questionnaire with application to the 1999-2006 NHANES questionnaire. Am J Epidemiol. 2013; 177(11):1199-208. [PubMed: 23595007]

34. Csizmadi I, Neilson HK, Kopciuk KA, Khandwala F, Liu A, Friedenreich CM, et al. The Sedentary Time and Activity Reporting Questionnaire (STAR-Q): Reliability and Validity Against Doubly Labeled Water and 7-Day Activity Diaries. Am J Epidemiol. 2014; 180(4):424-35. [PubMed: 25038920]

35. Chastin SF, Culhane B, Dall PM. Comparison of self-reported measure of sitting time (IPAQ) with objective measurement (activPAL). Physiol Meas. 2014; 35(11):2319-28. [PubMed: 25341050]

36. Gomersall SR, Pavey TG, Clark BK, Jasman A, Brown WJ. Validity of a Self-Report Recall Tool for Estimating Sedentary Behavior in Adults. J Phys Act Health. 2015; 12(11):1485-91. [PubMed: 26024378]

37. Jeran S, Steinbrecher A, Pischon T. Prediction of activity-related energy expenditure using accelerometer-derived physical activity under free-living conditions: a systematic review. Int J Obes (Lond). 2016; 40(8):1187-97. [PubMed: 27163747]

38. Kim Y, Barry VW, Kang M. Validation of the ActiGraph GT3X and activPAL Accelerometers for the Assessment of Sedentary Behavior. Meas Phys Edu Exerc Sci. 2015; 19(3):125-37.

39. Bai J, Di C, Xiao L, Evenson KR, LaCroix AZ, Crainiceanu CM, et al. An Activity Index for Raw Accelerometry Data and Its Comparison with Other Activity Metrics. PLoS One. 2016; 11(8):e0160644. [PubMed: 27513333]

40. Spiegelman D, Schneeweiss S, McDermott A. Measurement Error Correction for Logistic Regression Models with an "Alloyed Gold Standard”. Am J Epidemiol. 1997; 145(2):184-96. [PubMed: 9006315]

41. Ferrari P, Friedenreich C, Matthews CE. The Role of Measurement Error in Estimating Levels of Physical Activity. Am J Epidemiol. 2007; 166(7):832-40. [PubMed: 17670910] 


\section{Measurement Schedule (for group 1)}

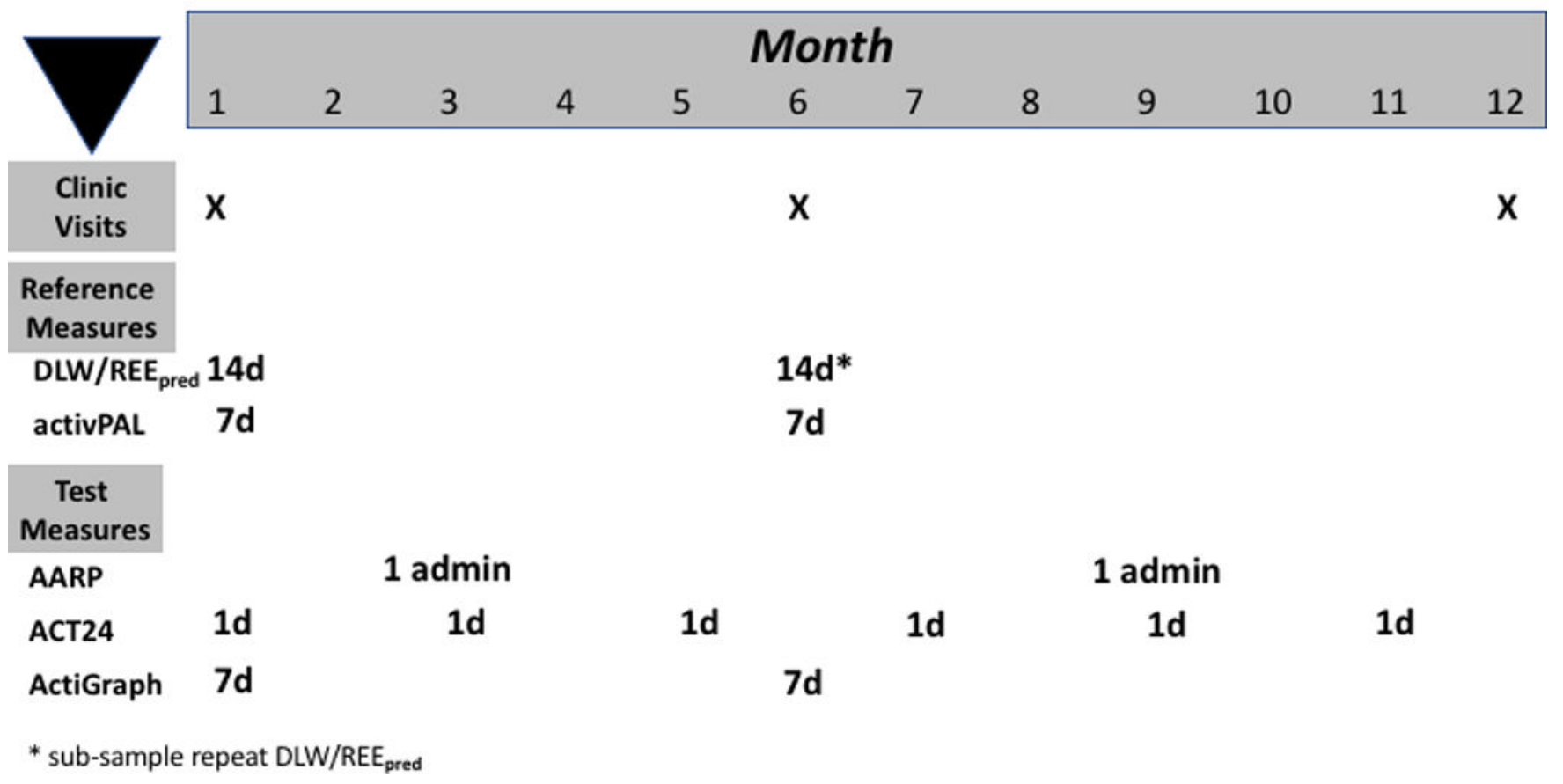

Figure 1.

Measurement schedule over 12-months for group one participants, IDATA Study, Pittsburgh, PA, 2012-2013 

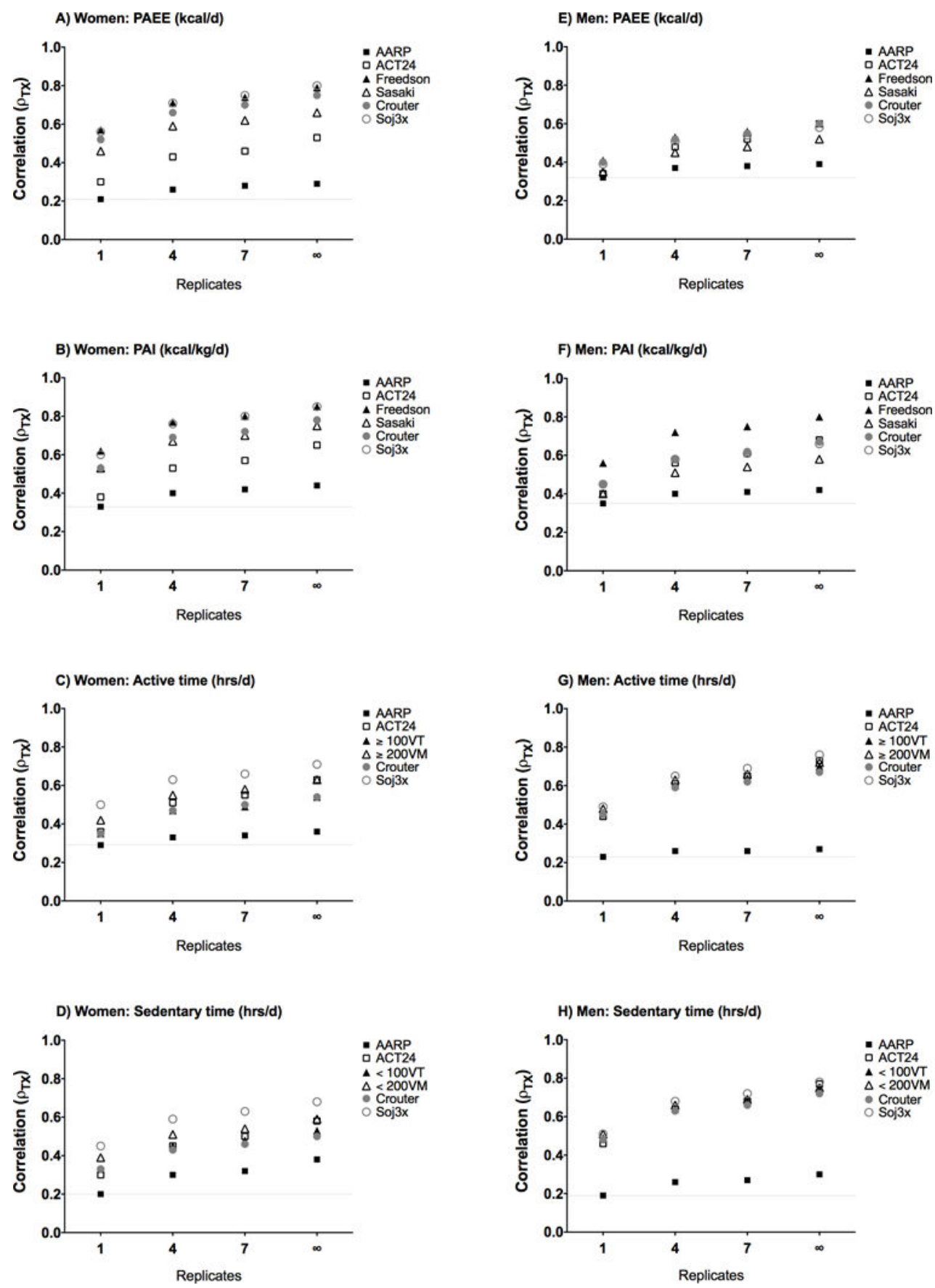

Figure 2.

Correlations for Each Test Instrument, by Sex and Number of Replicate Measures, IDATA Study, Pittsburgh, PA, 2012-2013

References measures for PAEE and PAI were from DLW

References measures for Active and Sedentary time were from activPAL 

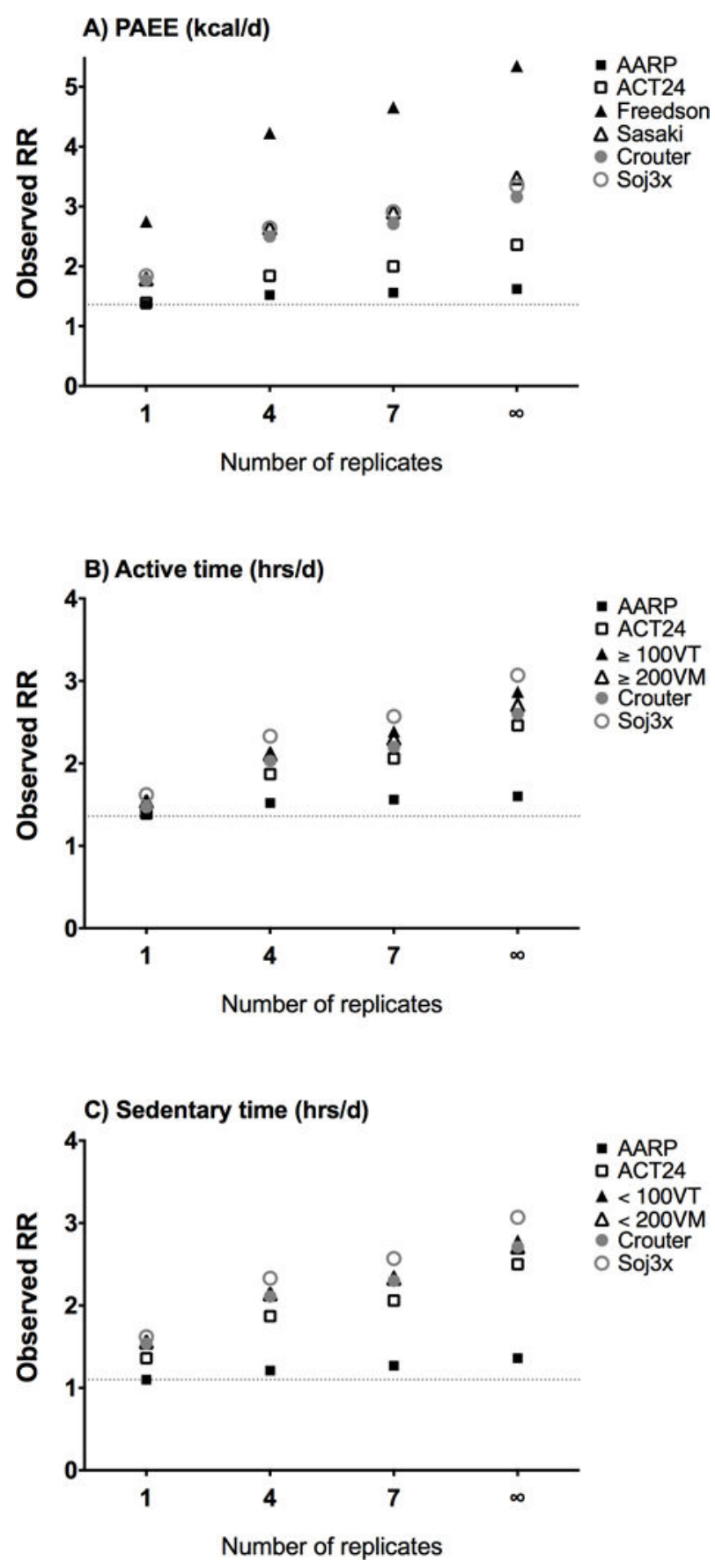

Figure 3.

Relative Risk Observed with Measurement Error for True Association of 4.0 for Each Test Instrument, by Number of Replicate Measures, IDATA Study, Pittsburgh, PA, 2012-201 


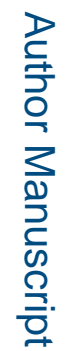

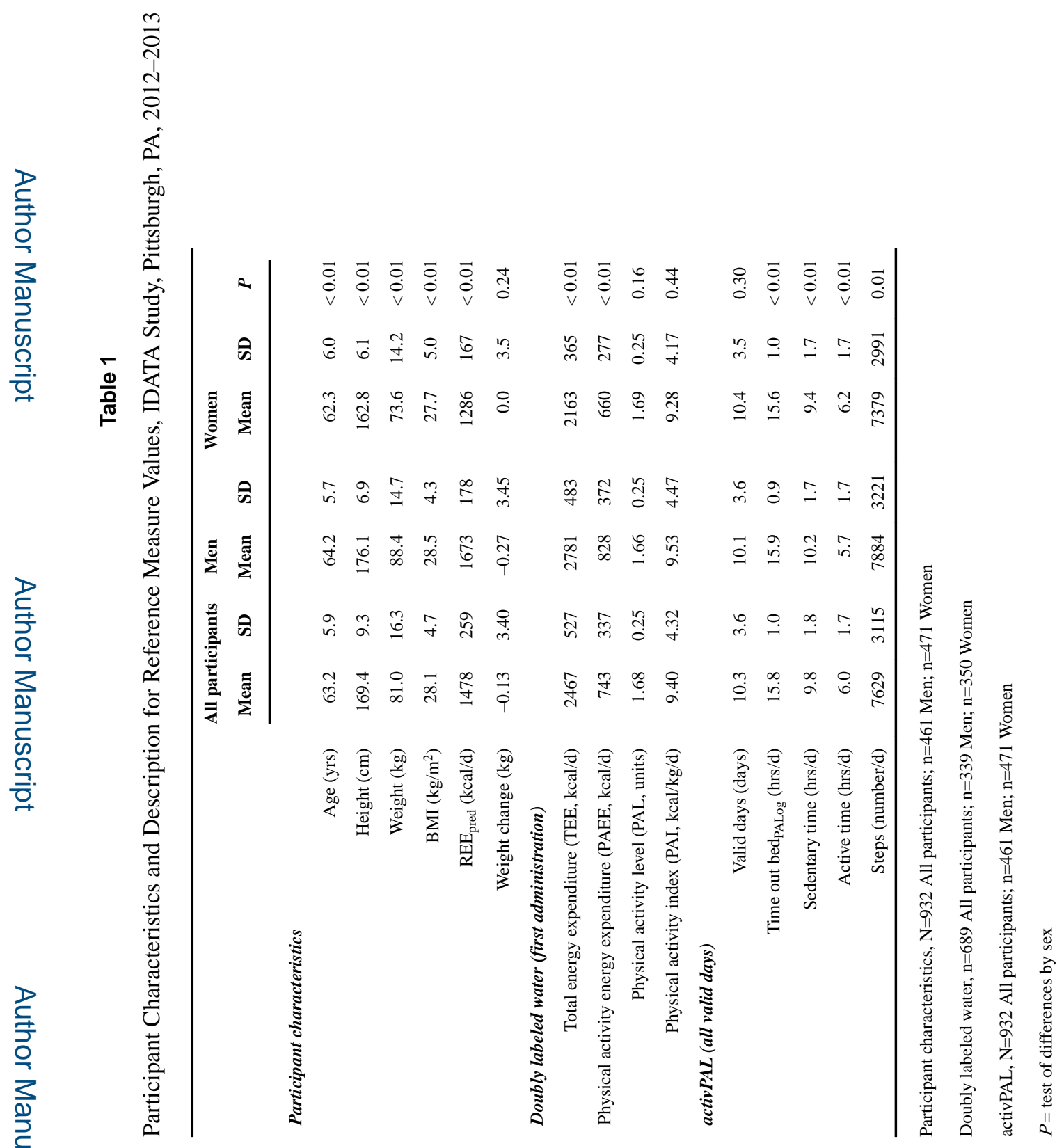




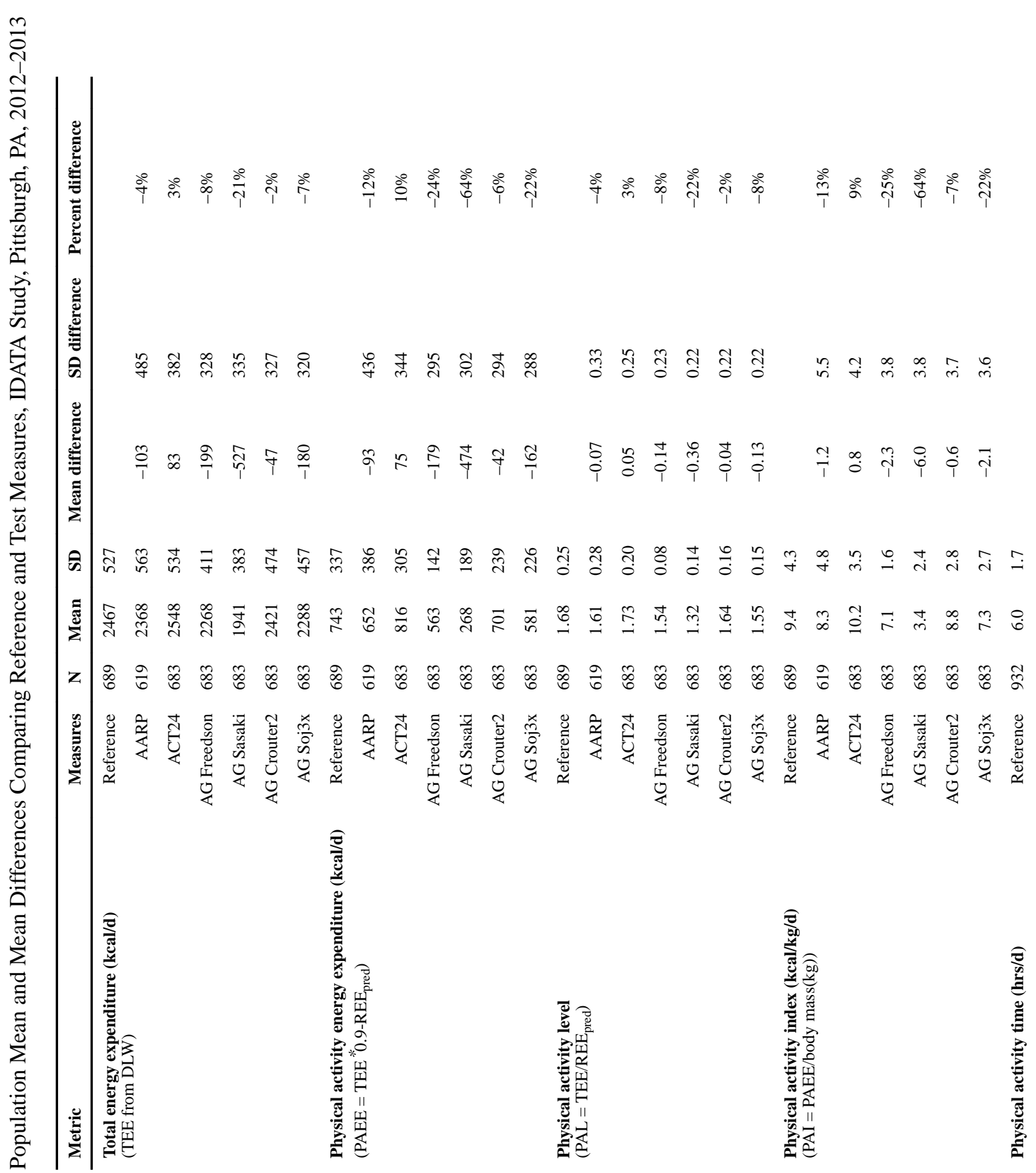


Matthews et al.

Page 19

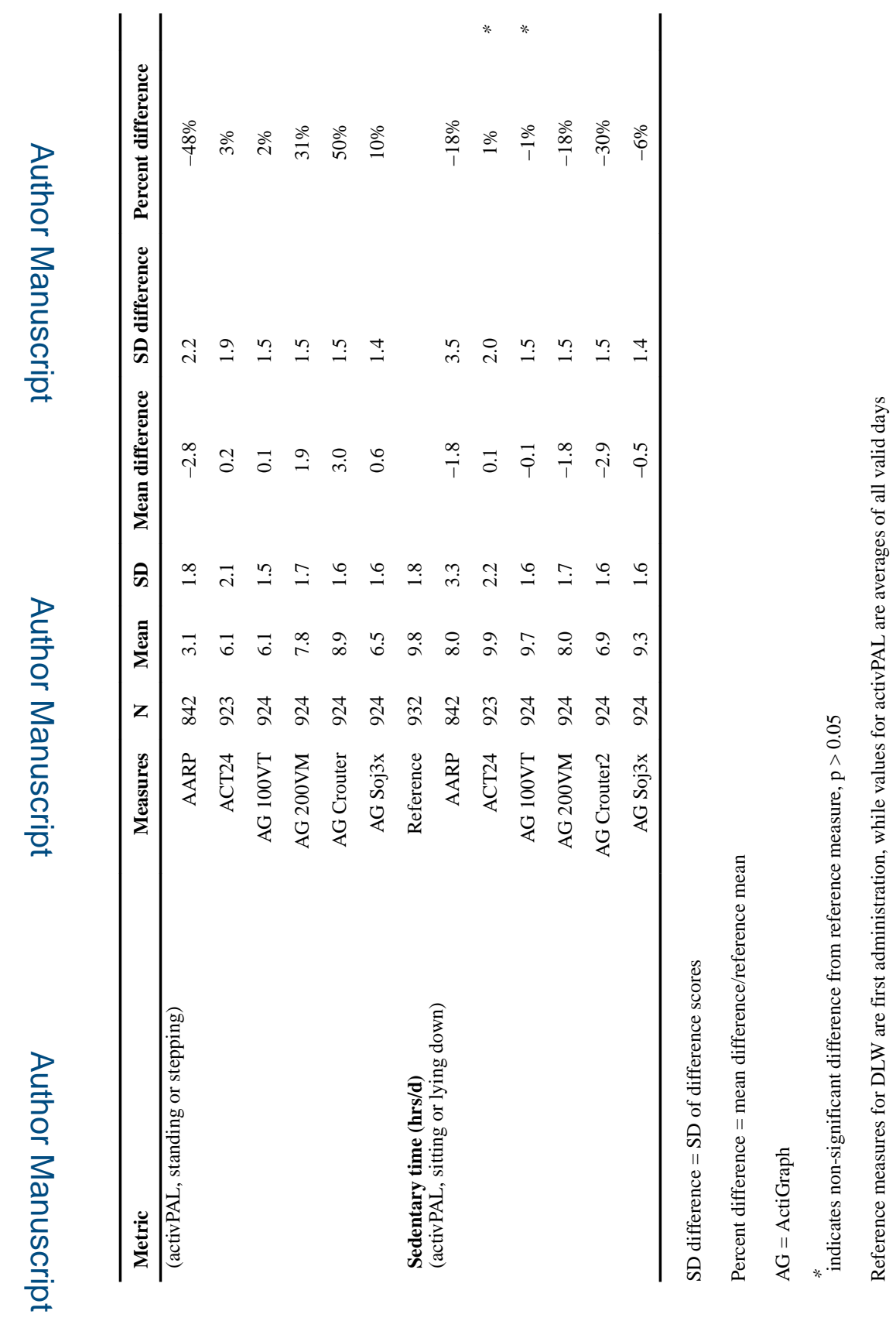

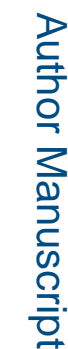

Med Sci Sports Exerc. Author manuscript; available in PMC 2019 February 01. 


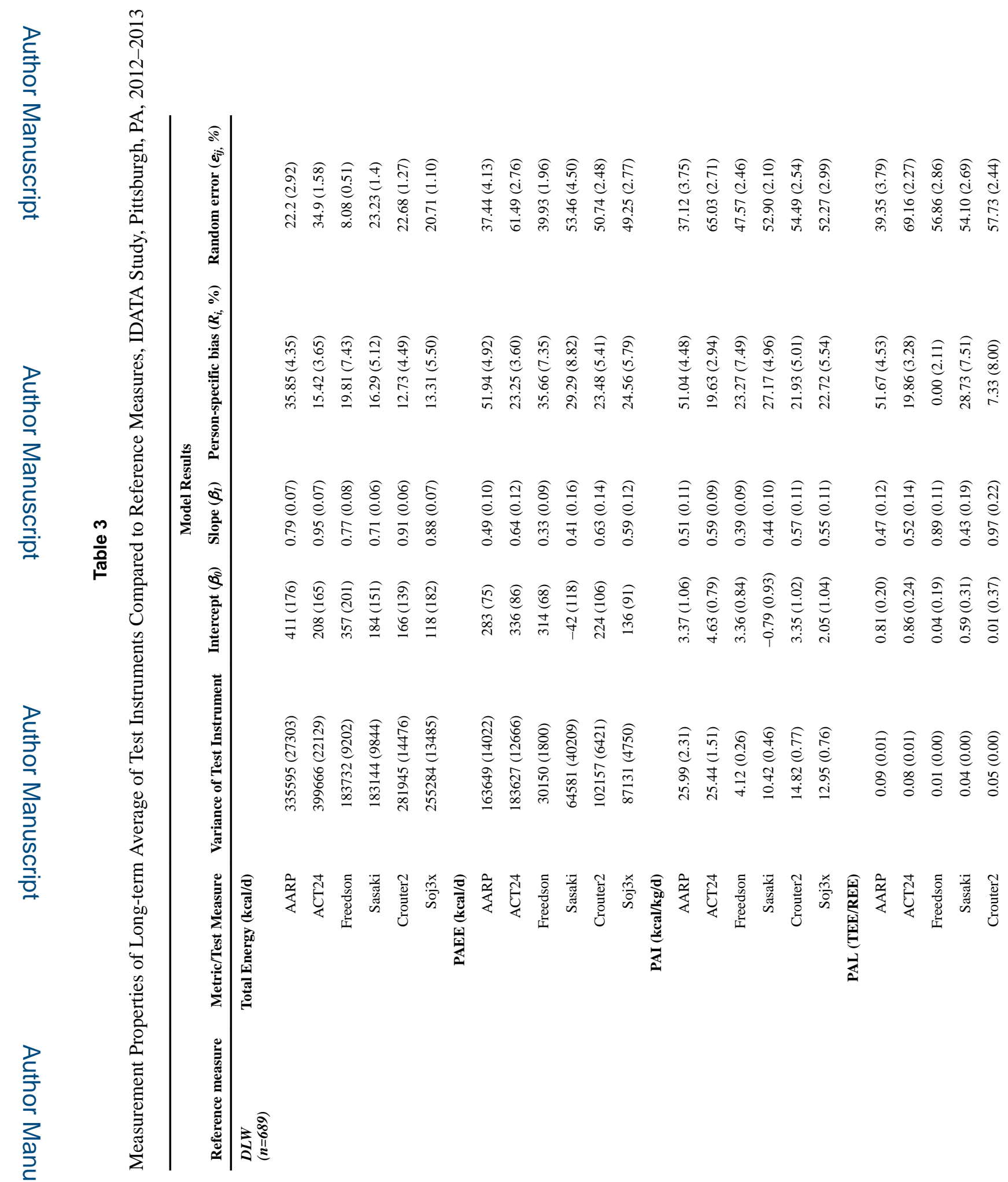

Med Sci Sports Exerc. Author manuscript; available in PMC 2019 February 01. 
Matthews et al.

Page 21

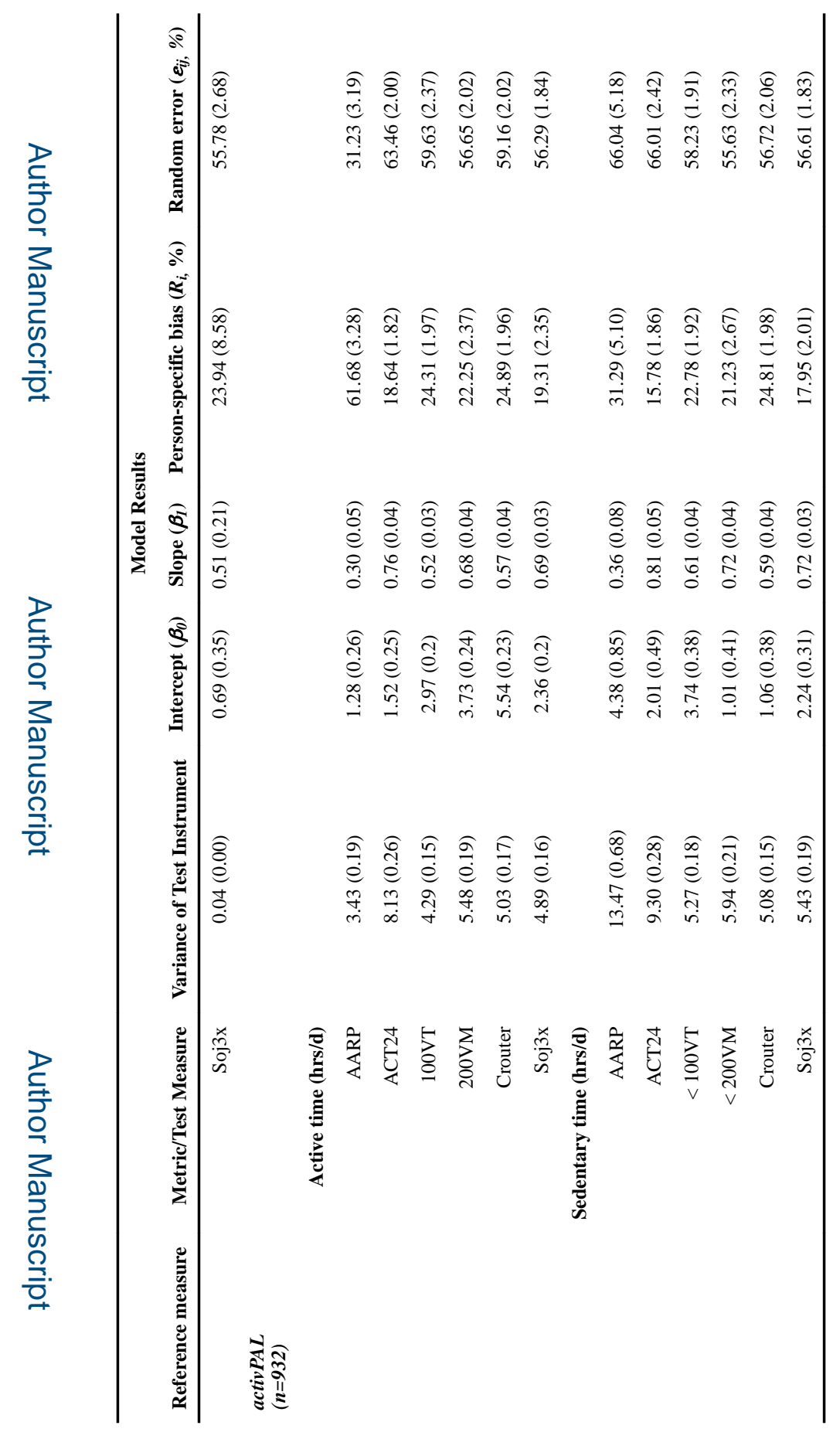

로을

Med Sci Sports Exerc. Author manuscript; available in PMC 2019 February 01. 